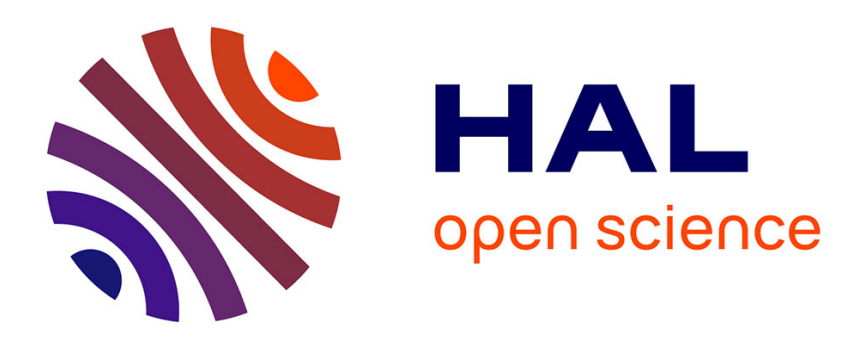

\title{
Les manuscrits de Ferdinand de Saussure déposés à la Bibliothèque de Genève en 2019
}

\author{
Pierre-Yves Testenoire
}

\section{To cite this version:}

Pierre-Yves Testenoire. Les manuscrits de Ferdinand de Saussure déposés à la Bibliothèque de Genève en 2019. Cahiers Ferdinand de Saussure, 2021, 73 (2020), pp.235-252. hal-03502198

\section{HAL Id: hal-03502198 \\ https://hal.science/hal-03502198}

Submitted on 24 Dec 2021

HAL is a multi-disciplinary open access archive for the deposit and dissemination of scientific research documents, whether they are published or not. The documents may come from teaching and research institutions in France or abroad, or from public or private research centers.
L'archive ouverte pluridisciplinaire HAL, est destinée au dépôt et à la diffusion de documents scientifiques de niveau recherche, publiés ou non, émanant des établissements d'enseignement et de recherche français ou étrangers, des laboratoires publics ou privés. 


\title{
LES MANUSCRITS DE FERDINAND DE SAUSSURE DÉPOSÉS À LA BIBLIOTHEQUE DE GENÈVE EN 2019
}

\author{
Pierre-Yves Testenoire
}

La Bibliothèque de Genève (BGE) a reçu en dépôt, en 2019, un lot de notes autographes de Ferdinand de Saussure. Après les premiers dons de manuscrits par les fils du linguiste, Jacques et Raymond de Saussure, dans les années 1950 et $1960^{1}$ et après l'arrivée en 1996 du fonds de manuscrits découverts dans l'orangerie de la propriété genevoise de la famille, ce dépôt constitue la troisième arrivée importante de manuscrits de travail de Saussure. Ce troisième dépôt n'a toutefois pas la même ampleur que les précédents : alors que le premier fonds (Ms. fr. 3951-3970) comprend environ 7000 feuillets et que celui de 1996 (Arch. de Saussure 366-388) compte plus de 5000 feuillets, le lot déposé en 2019 représente environ 400 feuillets ${ }^{2}$.

Ces manuscrits ont été remis à la Bibliothèque de Genève par la Fondation CharlesFrédéric Necker en même temps que d'autres archives intéressant l'histoire de la famille Necker. Ils sont classés sous la cote Arch. Necker 13 et ils sont répartis dans dix enveloppes.

Ces notes ont été classées et décrites par Robert Godel : sur chaque enveloppe figure une description de leur contenu de la main de Godel. Ces descriptions, au stylo bleu, sont similaires à celles que l'on trouve sur les enveloppes du fonds déposé dans les années 1950 et qui ont été publiées dans le numéro 17 des Cahiers Ferdinand de Saussure (Godel 1960). Il est donc évident que le classement de ces nouveaux manuscrits a été fait par Robert Godel à Vufflens en même temps que celui de ceux qui avaient été donnés en 1958 et qui forment aujourd'hui les cotes Ms. fr. 3952-3969. Pourquoi ces manuscrits ont-ils été séparés de ceux qui avaient été donnés à la BGE ? Nous l'ignorons. Sur l'historique de la conservation de ces papiers, nous ne disposons que des informations aimablement fournies par Alfred Necker, président de la fondation Charles-Frédéric Necker :

Ces manuscrits se trouvaient dans les archives de famille au moment du décès de mon grand-père, feu Henry Necker, survenu en mars 1947 ; ce dernier était le fils d'Hortense de Senarclens, épouse de Frédéric Necker : la sœur d'Hortense était Amélie de Senarclens épouse de Jules Faesch : leur fille Marie-Eugénie a épousé Ferdinand de Saussure (1857-1913) le célèbre linguiste.

Le berceau de la famille de Senarclens était le Château de Vufflens, tout près de Morges (Vaud) : après le décès de Henriette de Senarclens, née Macaire, dans les années 1895, ce château est demeuré en indivision de famille pendant environ 30 ans : ce n'est que dans les années 1924-25 que le partage successoral a pu intervenir. Le château, avec ses dépendances a été attribué à la branche Faesch-de Saussure, mais il a fallu trouver des compensations pour les autres branches. Henri Necker, étant très attaché à Vufflens où il avait passé une partie de son enfance, mais, étant déjà installé dans une maison de famille à Satigny, il reçoit (pour des considérations sentimentales), un certain nombres de «souvenirs » de Vufflens notamment : des portraits des familles de Senarclens et alliées, meubles, etc. ainsi qu'une petite partie des archives concernant l'histoire de Vufflens (certains documents datant du Moyen-Age) lesquels ont été déposées il y a quelques années aux Archives Cantonales vaudoises ainsi que le lot d'archives concernant Ferdinand de Saussure, dont il est question.

\footnotetext{
${ }^{1}$ Les dons de manuscrits autographes effectués entre 1955 et 1960 forment les cotes Ms. fr. 3951-3970 de la BGE, qui ont été complétées en 1988 par l'acquisition des manuscrits Ms. fr. 3974-3974a.

${ }^{2}$ Quelques autres textes autographes de Saussure figurent sous d'autres cotes à la Bibliothèque de Genève. Aux manuscrits genevois, il faut ajouter environ 650 feuillets autographes conservés à Harvard (v. D’Ottavi 2014) ainsi que des textes conservés dans les archives d'autres savants : par exemple dans le fonds Antoine Meillet du Collège de France (Amacker \& Bouquet 1988), dans le fonds Louis Havet de la BNF (Pinault 2012), dans celui de Louis Duvau à Saumur (Décimo 2012) ou dans celui de Charles Bally à Genève (Pesini 2010 ; 2012).
} 
Depuis lors ce lot a été conservé «religieusement» avec l'ensemble des archives de famille à Satigny : après le décès de Reyne Necker de Candolle en 1958 (ma grand-mère), ces archives avaient été attribuées à une fondation de famille dénommée «Fondation Henry Necker » : à la suite de changement de législation cette fondation a été dissoute en 2018 et l'ensemble du fonds a été transféré à la nouvelle «Fondation Charles-Frédéric Necker» (en souvenir du premier Necker arrivé à Genève du nord de l'Allemagne en 1724 et promu professeur de droit germanique à l'Académie de Genève) laquelle est reconnue d'intérêt public, et j'en suis, à ce jour, le président ${ }^{3}$.

Soixante ans après le don de 1958, les 400 feuillets rejoignent donc à la Bibliothèque de Genève les manuscrits dont ils ont été séparés. Ce nouveau lot de manuscrits est constitué en majorité de notes de travail de Saussure, auxquelles s'ajoute un peu de correspondance. Ces documents portent principalement sur la langue grecque. Ils peuvent être rapprochés des manuscrits déjà connus de la cote Ms. fr. 3952 et des notes éparses de linguistique grecque réunies sous les cotes AdS 382 et 383.

Nous présentons ci-dessous le contenu détaillé des 10 enveloppes réunies sous la cote Arch. Necker 13 en les réunissant sous cinq grandes entrées thématiques. Lorsque cela est possible, nous mettons ces nouveaux manuscrits en relation avec les publications du linguiste et avec les autres manuscrits saussuriens conservés à la Bibliothèque de Genève. L'intitulé des cotes qui figure entre guillemets correspond au titre écrit par Robert Godel sur chaque enveloppe.

Des images numériques de ces manuscrits sont disponibles sur le site de la Bibliothèque de Genève : https://archives.bge-geneve.ch/archive/fonds/necker_famille

\section{Etymologies}

Arch. Necker 13/1, «Etymologie » (22 f.)

Dans cette enveloppe ont été réunies plusieurs notes sur l'étymologie de mots grecs ou gotiques. La plupart d'entre elles ont été rédigées lors des dernières années du séjour de Saussure à Paris. Certaines ont été publiées dans la rubrique «Variété » des Mémoires de la Société de linguistique de Paris [M.S.L] dont Saussure a la charge de 1881 à 1891 ; d'autres non. On trouve aussi une note plus tardive sur l'étymologie d'ỏ $\lambda$ oxí rédigée en 1905.

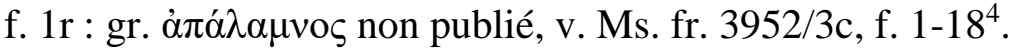

f. $1 \mathrm{v}-2$ : blanches.

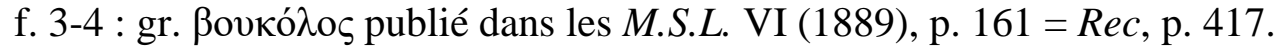

f. 5-6 : gr. ỏкрvóєıৎ "à composer en pages à la suite de l'article sur "Gothique wilwan" » qui paraît dans les M.S.L. VI (1889). La note sur ỏкpvóeıৎ sera finalement publiée sous une forme plus courte dans les M.S.L VII (1992), p. $88=$ Rec, p. 456.

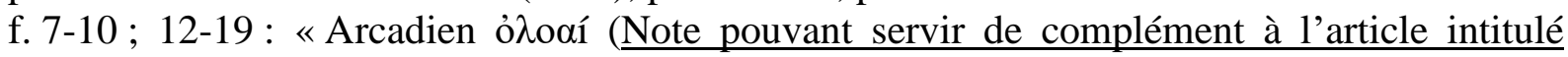

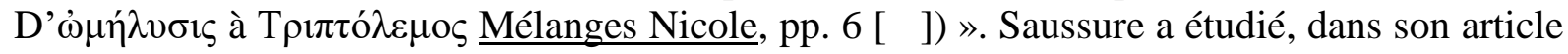

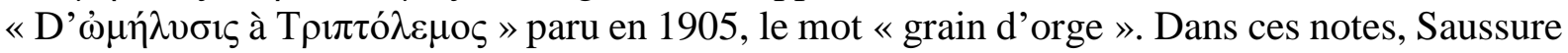

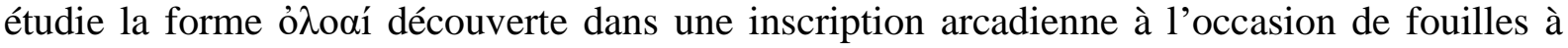

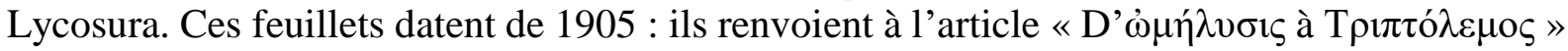
alors en cours de parution ${ }^{5}$. L'un des feuillets (f. 14) contient des remarques sur l'emploi du

\footnotetext{
${ }^{3}$ Alfred Necker, communication personnelle, le 27 novembre 2020.

${ }^{4}$ Godel (1960 : 9) note que «Saussure a exposé cette étymologie ( $\dot{\alpha} \pi-\dot{\alpha} \lambda \alpha-\mu \nu$ os “celui qui s'est égaré") dans un entretien du 26 novembre 1910 avec M. L. Gautier, qui m'a communiqué ses notes ».

${ }^{5}$ Ces notes sont à mettre en relation avec Ms. fr. 3955/3, f. 1-7 et avec les lettres à Charles Bally et Antoine Meillet de septembre et octobre 1905 (Benveniste 1964 : 102-103 et Amacker 1994 : 98-101).
} 
mot «montagne » relevé dans la Feuille des avis officiels du Canton de Vaud de fin août-début septembre 1905. Cette note est à mettre en relation avec le travail sur les noms géographiques de la Suisse romande (Ms. fr. 3956).

f. $11 \mathrm{v}$ : un exercice de sanskrit à l'intention d'étudiants ${ }^{6}$.

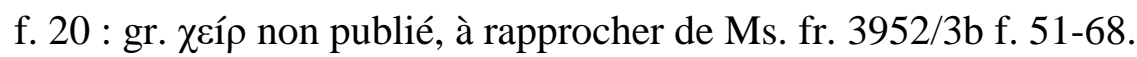

f. 21 : gr. $\pi \rho \varepsilon ́ \pi \omega$ et lat. corpus. Note écrite sur une lettre du Comte de Charencey du 12 mai 1885, reçue par Saussure dans le cadre de son activité de secrétaire adjoint de la SLP. Le 20 février 1886 Saussure fait une communication à la SLP sur «l'étymologie du verbe $\pi \rho \varepsilon ́ \pi \omega$; il conclut à une parenté avec le latin corpus » (BSL $6:$ LXXVI) ${ }^{7}$.

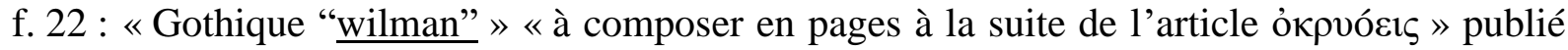
dans les M.S.L. VI (1889), p. $358=$ Rec, p. 434.

\section{Linguistique grecque et latine}

Arch. Necker 13/2-3, « Notes diverses sur le grec » (95f.)

f. 2-4 : notes de phonétique grecque, sur les semi-consonnes $j$ et $w$.

f. 5-7: « Notes grecques ».

f. 8 : brouillon de lettre à Louis Havet (Versoix, 17 mars sans millésime).

f. 9-11 : aoriste gr. "̌ $\pi \varepsilon \sigma o v$ dont Saussure parle dans son brouillon de lettre à Havet.

f. 12-20bis : notes de cours sur les dialectes grecs, v. f. 12 «Dialect. grecs 25 févr. »; f. 18 « $\Delta 1 \alpha \lambda . \mu \varepsilon \rho \kappa \rho \varepsilon \delta 121 »$.

f. 21-31 : notes de phonétique grecque, vraisemblablement pour un cours.

f. 32-33: « aoristes du type ع̈ $\mu \varepsilon เ v \alpha »$.

f. 34-46: gr. $\pi \rho \varepsilon ́ \pi \omega$, v. supra.

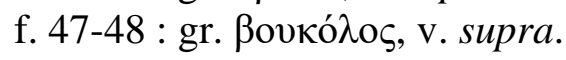

f. 49-59: blanches.

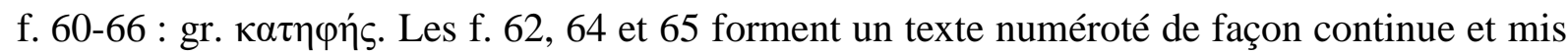
au propre. Un brouillon du f. 64 figure en AdS 382/6, f. 98.

f. 67-69; 71-73 : « la particule négative vๆ », v. aussi AdS 382/6, f. 99.

f. $70 ; 74 ; 76-78$ : «Composés remarquables non homériques ».

f. 75 : note sur les neutres gr. en $-\alpha-\varsigma$ et skr. en -i-s.

f. 79-80 : traduction de deux passages de l'Agamemnon d'Eschyle. Le f. 79 est à insérer dans l'édition de Mejia Quijano \& Restrepo Montoya (Saussure 2008) entre les pages 207 et 208 ; le f. 80 est à insérer page 225. Un brouillon de ce feuillet est conservé (AdS 372, f. 27) et transcrit dans Saussure $2011: 333$. Voir Annexe.

f. 81-87: relevés lexicaux grecs.

f. 88 : notes écrites sur un imprimé daté de 1905.

f. 89-95 : notes et schémas sur les tables d'Héraclée, inscription grecque étudiée par Saussure dans le cadre de ses cours entre 1894 et 1896 (Murano 2015). Ces notes sont à mettre en relation avec Ms. fr. 3957/7, f. 12-16 et avec les notes conservées dans le fonds Charles Bally : Ms. fr. $5131 / 1-2^{8}$.

f. $95 \mathrm{v}$ : notes sur les tables d'Héraclée de la main de Charles Bally.

\footnotetext{
${ }^{6}$ V. d'autres exemples dans AdS 387/8 et AdS 383/2, f. 54.

${ }^{7}$ Des notes préparatoires à cette communication sur $\pi \rho \varepsilon ́ \pi \omega$ se trouvent à la BGE, AdS 383/8, f. 14, 383/9, f. 21, f. 53 et 383/10, f. 58. Sur les activités de Saussure en tant que secrétaire adjoint de la SLP et ses échanges avec le Comte de Charencey dans ce cadre, v. Testenoire 2018.

${ }^{8}$ Sur le cours dans lequel s'inscrit l'étude des tables d'Héraclée, v. Murano 2017 : 58-69.
} 
Sous cette cote figurent un texte continu de 24 feuillets paginés par Saussure de 1 à 44, ainsi qu'une enveloppe sur laquelle est écrit « $\varphi$ on. Grécolat. ». Le texte porte sur le traitement des vélaires indo-européennes en grec et en latin. La distinction de deux séries de gutturales indo-européennes a été établie au début des années 1870 par Graziado Isaia Ascoli et August Fick. Comme il le fait dans son Mémoire, Saussure note $k_{l}, g_{l}, g_{l} h$ les gutturales avant (palatales) et $k_{2}, g_{2}, g_{2} h$ les gutturales arrière (vélaires) ${ }^{9}$. La question des gutturales indoeuropéennes est alors un point débattu, en particulier la distinction entre vélaires pures et labiovélaires. Voici son traitement détaillé dans ces notes :

f. $1: k_{2}$ devant voyelle

f. $2-4 \mathrm{v}:$ : Le traitement de $\mathrm{k}_{2}$ devant voyelle dans les dialectes grecs »

f. $2-3 \mathrm{r}$ : «ce qu'on entend par les dialectes ${ }^{10}$

f. $4 \mathrm{v}-5 \mathrm{r}: \mathrm{k}_{1}$ et $\mathrm{k}_{2}$ en latin

f. $5 v-6:$ : $\mathrm{k}_{2}$ devant consonne. Premièrement en latin »

f. $6: \ll \mathrm{k}_{2}$ devant consonne en grec »

f. 7-8 : « $\mathrm{k}_{2}$ rendu par $\mathrm{k}$ sans appendice labial»

f. $9-10: \ll g_{1} »$

f. 11-14: « $\mathrm{g}_{2}$ en grec devant voyelle »

f. 15-17: « $\mathrm{g}_{2}<$ devant voyelle> dans les dialectes grecs »

f. $18:$ : $\mathrm{g}_{2}$ devant consonne en latin »

f. 18-19r : « $\mathrm{g}_{2}$ devant consonne en grec »

f. 19-23r: « $\mathrm{g}_{2}$ rendu par g pur»

f. $23: \ll \mathrm{g}_{1} \mathrm{~h} \& \mathrm{~g}_{2} \mathrm{~h} »$

f. 24 : enveloppe sur laquelle est écrit « $\varphi$ on. Grécolat. ».

Ces manuscrits sont, de toute évidence, les notes d'un cours sur la phonétique grecque et latine. Dans la liste des enseignements donnés par Saussure à Paris puis à Genève, deux intitulés peuvent correspondre à ces notes :

- Les conférences sur la « Grammaire comparée du grec et du latin » que Saussure donne à l'Ecole des Hautes Etudes en 1887-1888 et 1888-1889, les conférences de la seconde année ayant «été la continuation des conférences de l'année précédente ${ }^{11}$.

- Le cours «Phonétique du grec et du latin » que Saussure donne les deux premières années de son enseignement à Genève (1891-1893).

$\mathrm{Si}$, à ce jour, aucune note d'auditeurs n'a été retrouvée pour le premier cours, le second cours a été pris en note par Albert Sechehaye dont les cahiers sont conservés à la BGE (non catalogué 2002/026) ${ }^{12}$. Or, le sommaire du cours dans les cahiers de Sechehaye ne correspond pas aux notes de Saussure. L'ordre suivi pour le traitement des vélaires dans le cours de 1891 tel que Sechehaye l'a consigné - $k_{1}, g_{1}, g_{1} h, k_{2}, g_{2}, g_{2} h$-n'est pas celui des notes de Saussure. En outre, les analyses contenues dans les manuscrits de la cote Arch. Necker 13/4 sont beaucoup plus détaillées que ce que l'on trouve dans les cahiers de Sechehaye. Ces notes s'adressent à un auditoire plus avancé comme l'était celui de Saussure lors de ses dernières années à l'Ecole des Hautes Etudes. Il y a donc tout lieu de penser que ces manuscrits sont des notes pour les leçons de phonétique du cours de grammaire comparée de 1887-1889.

Ce cours revêt dans l'itinéraire de Saussure une importance capitale. Non seulement il constitue le premier élargissement de son enseignement à Paris hors du domaine germanique mais il réunit des auditeurs, dont plusieurs deviendront d'éminents linguistes : Paul Boyer,

\footnotetext{
${ }^{9}$ Sur l'histoire de cette notation, v. Pesini 2010 et Saussure $2018: 154$.

${ }^{10}$ Sur la conception des dialectes chez Saussure, v. Murano 2017.

${ }^{11} \mathrm{~V}$. le rapport de ce cours dans l'Annuaire de l'EPHE (Fleury 1964 : 66).

${ }^{12}$ Alessandro Chidichimo a publié les premières pages de ces cahiers : Sechehaye 2009.
} 
Georges Dottin, Sylvain Levi, Antoine Meillet, Georges Möhl ${ }^{13}$. Cette nouvelle génération de comparatistes issus de l'EPHE forme ce qu'on a pu appeler l' « école française de linguistique » ou l'« école de Paris ». Le cours de grammaire comparée de 1887-1889 joue, à titre rétrospectif, un rôle de creuset dans l'imaginaire de la constitution de cette école saussurienne à Paris (Savatovsky 1999 ; 2003).

Ce cours est constitué de deux séries de leçons sur les deux années : l'une consacrée à la phonétique, l'autre à la morphologie mais « la phonétique seule a pu être achevée ; l'exposé de la morphologie est demeuré malheureusement incomplet faute de temps » (Fleury $1964: 66$ ). Pour les leçons de morphologie, nous connaissions déjà les manuscrits Ms. fr. 3970/c édités par Angeli \& Vallini (Saussure 1990). En ce qui concerne les leçons de phonétique, le texte continu d'Arch. Necker 13/4 semble appartenir au même ensemble que les notes conservées sous les cotes Ms. fr. 3952/2c et Ms. fr. 3952/2d. Robert Godel, au moment du classement de ces papiers, avait fait l'hypothèse qu'ils correspondaient au cours de phonétique grecque et latine de 1892-1893. La conservation au milieu de ces notes d'une lettre de Paul Boyer (Ms. fr. 3952/2c, f. 39) portant sur le contenu du cours corrobore l'attribution à la période 1887$1889^{14}$. Enfin, la cote AdS 383 rassemble des notes de ce même cours, en particulier le texte continu du f. 144 au f. 193 qui porte sur la phonétique grecque et latine. Mis bout à bout, ce sont plus de 150 feuillets qui correspondent aux leçons de phonétique du cours de grammaire comparée de l'Ecole des Hautes Etudes, un puzzle que le dépôt des manuscrits de 2019 contribue à agencer.

\section{Correspondance et brouillons de lettres}

Arch. Necker 13/5, « Divers » (61f.)

Dans cette enveloppe sont réunis des lettres reçues par Saussure, divers brouillons de lettres adressés à plusieurs correspondants et quelques papiers et imprimés épars. Dans cet ensemble, on relève :

f. $2-4 ; 19 \mathrm{v}$ : brouillons de lettre à un correspondant non identifié auquel Saussure demande des renseignements sur les éditions d'Homère pour un travail statistique sur le digamma homérique. L'intérêt pour cette question fait suite à une lettre de Charles Bally du 15 mai 1902 (AdS 366, f. 6-7). La réponse de Saussure (AdS 366, f. 8-10 et Ms. fr. 5134, f. 5-10) ainsi que plusieurs notes (Ms. fr. 3954, f. 89-95, AdS 383/2, f. 1-15) portent sur ce travail statistique.

f. 5-16: blanches.

f. 17-20;22-23 : notes sur la phonologie et sur la phonétique indo-européenne ${ }^{15}$.

f. 24-26: brouillon de lettre adressé à Max Niedermann, où Saussure commente et critique ses «Notes d'étymologie latine » publiées en 1902 dans les Mélanges linguistiques offerts à Antoine Meillet. La lettre ne sera pas envoyée si l'on en croit la première lettre de Saussure à Niedermann qui a été retrouvée. Cellec-ci date de 1904 et commence en ces termes : « ce n'est là qu'un des nombreux ouvrages dont je suis redevable à votre attention pour un vieux collègue.

\footnotetext{
${ }^{13}$ Pour la liste exhaustive des auditeurs et les données prosopographiques les concernant, v. Décimo 2014.

${ }^{14} \mathrm{La}$ lettre de Boyer contient une question «à propos de la dernière leçon sur la phonétique syntactique »: l'élève se demande «s'il n'y a pas une légère inexactitude dans [un] passage de la dernière leçon ». Elle est à rapprocher de la première de lettre de Saussure à Meillet récemment découverte (Testenoire 2015) qui est une réponse à une question-objection portant sur le même cours. Ces documents nous éclairent sur la relation pédagogique que le jeune maître de conférences avait instaurée à Paris avec ses étudiants.

${ }^{15}$ L'un des feuillets (f. 22) date du début de la période parisienne : après avoir critiqué la théorie d'Osthoff sur la racine du sanskrit sīvyati «coudre », Saussure renvoie à sa propre communication du 28 mai 1881, théorie « donnée il y a deux ans à Brugmann, elle a fait depuis le sujet d'une communication à la Société de Linguistique de Paris ».
} 
Les remerciements que je vous dois remontent, non à des semaines, mais à des années » (Piccini 2020 [2021]).

f. 27-28 : notes adressées à Charles Bally concernant ses travaux de linguistique grecque. f. 27-

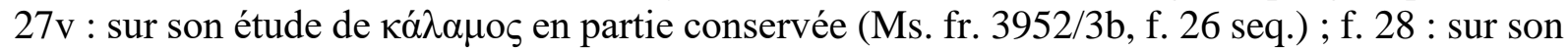

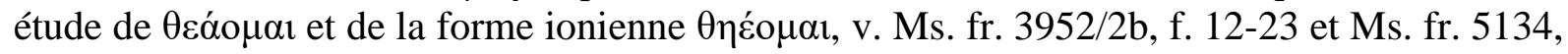
f. 11-13.

f. 29-30 : lettre du linguiste italien Francesco Lorenzo Pullé, datée du 18 septembre 1894, juste après le Congrès des orientalistes de Genève auquel il a participé.

f. 31-33 : brouillon de lettre à Louis Duvau daté de l'automne 1894. Saussure y revient sur le déroulement du Congrès des orientalistes de Genève et le conflit qui l'opposa à certains membres de la commission sur la transcription du sanskrit ${ }^{16}$.

f. 34 : brouillon de lettre à un correspondant inconnu pour la présentation de Charles Bally à la Société de linguistique de Paris ${ }^{17}$.

f. 35 : note sur le suffixe grec - $\eta$ vó

f. 36-37 : lettre de Charles Seitz, doyen de la faculté des Lettres de l'Université de Genève, datée du 9 novembre 1907.

f. $41-42 ; 57 \mathrm{v}-58$ : notes sur l'actualité, et notamment sur la guerre gréco-turque de $1897^{18}$.

f. 44-55 : deux tirés à part : une nécrologie de Henri de Saussure, père du linguiste décédé en 1905 et la première publication de F. de Saussure : «suffixe T » (1877).

f. 59-61 : notes sur les courses de chevaux et comptes de parties de poker datant de la période parisienne, similaires à ce que l'on trouve sous AdS 383/3, f. 98-101.

\section{Vers saturniens}

Arch. Necker 13/6 : «Vers saturniens » (41f.)

Cette enveloppe contient un ensemble de 41 feuillets sur les vers saturniens. Ces manuscrits datent du premier semestre 1906, au moment où Saussure travaille sur la versification de ce vers latin archaïque (Testenoire 2013: 52 sq.). Le linguiste y analyse l'explication métrique du vers saturnien proposée par Louis Havet dans sa thèse De Saturnio latinorum versu (1880). Saussure récuse l'explication d'Havet, « un vers iambique composé de deux membres comportant chacun trois temps marqués», ce qu'il nomme «la théorie métrique » (f. 7). Cette enveloppe est à rapprocher des manuscrits sur le saturnien de la période pré-anagrammatique (Ms. fr. 3962).

f. 1-15 : liasse de feuillets analysant la structure métrique du corpus de vers saturniens étudiés par Havet.

f. 16-23v : cahier de 8 feuillets où Saussure examine l'allitération dans les vers saturniens.

f. 24-31 : cahier de 8 feuillets où Saussure analyse la composition en « unités » lexicales des vers saturniens, ce qu'il appelle ailleurs le « schéma verbal ».

f. 32-33 : bi-feuillet sur les vers saturniens.

f. 34-41: cahier de 8 feuillets où Saussure analyse la structure métrique et verbale des saturniens.

\footnotetext{
${ }^{16}$ Sur cet incident, v. Amacker 2006 et Joseph 2012 : 402-409 et AdS 369/11.

${ }^{17}$ La formule d'adresse « Monsieur et cher maître » laisse supposer que le brouillon est adressé à Michel Bréal ou à Gaston Paris. Finalement, Charles Bally est élu à la Société de linguistique de Paris le 24 février 1900 sur présentation d'Antoine Meillet et de Ferdinand de Saussure.

${ }^{18}$ Ces notes sont à rapprocher de celles que l'on trouve sous la cote AdS 371/1-3.
} 


\section{Inscriptions phrygiennes}

Les quatre dernières enveloppes réunissent des notes de travail en lien avec l'article «Inscriptions phrygiennes » paru en 1898 dans l'ouvrage d'Ernest Chantre: Recherches archéologiques dans l'Asie Occidentale : Mission en Cappadoce, 1893-1894 = Rec, p. 542575. L'étude porte sur une inscription phrygienne découverte par Ernest Chantre à l'occasion de fouilles à Euyuk en 1893 et 1894. En septembre 1895, Saussure est sollicité par l'intermédiaire d'Alfred Boissier, assyriologue genevois, qui a participé à la mission Chantre de 1894. Le travail sur le déchiffrement et l'interprétation de cette inscription prend place en 1895 et 1896 (Marchese \& Murano 2015 : 98 sq.). Saussure identifie en phrygien un suffixe toponyme $-\bar{a} n$ qu'il rapproche du suffixe grec $-\eta v o ́ \varsigma$ des noms ethniques. Ce rapprochement

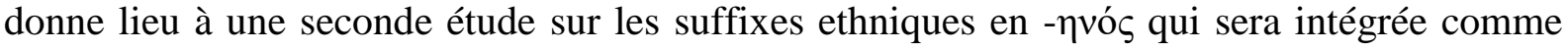
appendice à l'article «Inscriptions phrygiennes » de 1898. Les notes réunies dans les quatre enveloppes sont à mettre en relation avec les notes qui figurent sous les cotes Ms. fr. 3955/2, AdS 379/8 et, de façon éparse, AdS 382/4-7, en particulier f. 26-60 ; 118-121.

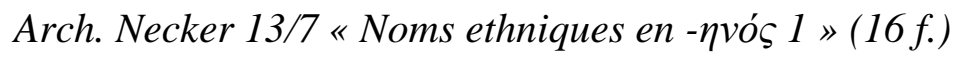

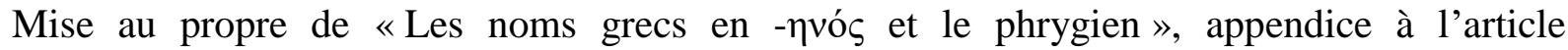
«Inscriptions phrygiennes » (Rec, p. 566-575). Le texte est numéroté de 1 à 16 par Saussure et signé. Les consignes de mise en page («pas d'alinéa! » «Verso ! ») indiquent que le texte est adressé à l'imprimeur.

Arch. Necker 13/8 «Noms ethniques en - $\eta$ vós $2 »(89$ f.)

f. 1-17 : cahier d'écolier sur les suffixes ethniques en -ๆvóc.

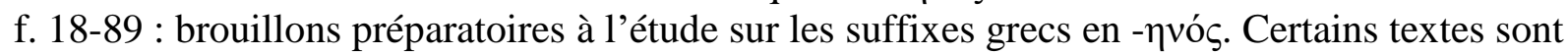
mis au propre.

f. 69 : brouillon écrit sur un faire-part de mariage daté de mars 1897.

f. 81-89 : dépouillement de sources : plusieurs feuillets sont de la main de l'épouse du linguiste, Marie de Saussure.

Arch. Necker 13/9 «Inscriptions phrygiennes $1 »(94 f$.

f. 1-13 : cahier d'écolier contenant des notes sur les inscriptions découvertes en Cappadoce par Ernest Chantre en 1893 et 1894.

f. 13-19: correspondance reçue en lien avec le travail sur les inscriptions découvertes en Cappadoce :

- f. 13-14: lettre de Joachim Menant, assyriologue et membre de l'Académie des Inscriptions et Belles Lettres, datée du 26 septembre $1895^{19}$.

- f. 15-18 : deux lettres d'Ernest Chantre, archéologue, datées du 20 mai et du 7 juillet 1896.

- f. 19-20 : copie des inscriptions phrygiennes envoyées par Alfred Boissier en septembre $1895^{20}$.

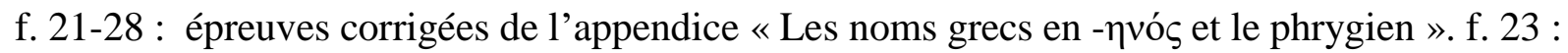

«Bon à tirer pour les six pages après consciencieuse correction. F. S. »

f. 29-42; 45-94 : notes et brouillons préparatoires à l'article « Inscriptions phrygiennes ».

\footnotetext{
${ }^{19}$ V. la réponse de Saussure à cette lettre et les échanges avec Joachim Menant (Saussure $2014: 237-240$ ).

${ }^{20}$ V. la lettre de Saussure à Alfred Boissier datée du 29 septembre 1895 (Saussure $2014: 236$ ).
} 
f. 43-44 : «Supplément aux questions indiquées dans ma lettre » dans lequel Saussure adresse cinq questions sur la reproduction des inscriptions. Alfred Boissier répond à chacune des questions.

f. 46v : «Histoire et comparaison des langues indo-européennes »: brouillon d'un texte de Saussure sur son enseignement paru dans une brochure de l'Université de Genève en 1896 (Chidichimo 2019). V. également Ms. fr. 3970/c, f. 61-62, AdS 369/10, f. 1 et AdS 383/13, f. 18-21.

f. 51v : brouillon écrit au verso d'un courrier administratif daté du 11 septembre 1895.

Arch. Necker 13/10 «Inscriptions phrygiennes $2 »(37 f$.

Mise au propre de l'article «Inscriptions phrygiennes » sans l'Appendice II « Les noms grecs

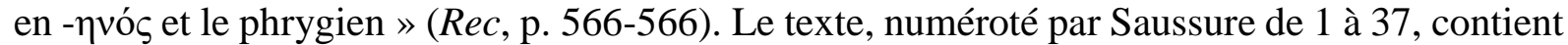
de nombreuses consignes adressées au typographe : « Ici le croquis $n^{\circ} 1$ !!! » (f. 3), «!! 1 ligne en blanc » (f. 8), «pas d'alinéa! » (f. 10), «!!! Toute cette note est à composer en petites lettres !! » (f. 19), etc.

Pierre-Yves Testenoire pierre-yves.testenoire@sorbonne-universite.fr Sorbonne Université, Faculté des Lettres INSPE de Paris, F-75016 Paris, France Université de Paris and Université Sorbonne Nouvelle, CNRS,Laboratoire d'histoire des théories linguistiques , F-75013 Paris, France 


\section{ANNEXE \\ TRADUCTION DE L'AGAMEMNON D'ESCHYLE \\ (ARCH. NECKER 13/2-3, F. 79-80)}

Les deux pages que nous éditons ici sont à insérer dans le dossier des manuscrits de Saussure sur l'Agamemnon d'Eschyle (Saussure 2008). Ce dossier est constitué d'une trentaine de feuillets, éparpillés sous différentes cotes de la Bibliothèque de Genève, contenant la traduction et le commentaire de la première pièce de l'Orestie. Il s'agit de notes préparatoires à un cours donné par Saussure à l'automne 1896 en remplacement de son collègue de langue et littérature grecques de l'Université de Genève, Jules Nicole. La traduction couvre de façon quasi-continue environ la première moitié de la pièce. Elle va du v. 280 au v. 876 dans les éditions modernes, ce qui correspond aux v. 265-843 dans les deux éditions qu'utilise Saussure : celles d'Hermann (1852) et de Schneidewin (1856). Quelques lacunes étaient toutefois à signaler : ce sont deux d'entre elles que les f. 79 et 80 de la cote Arch. Necker 13/23 viennent combler.

Les deux feuillets n'ont peut-être pas été isolés par hasard : ils correspondent, en effet, tous les deux à des passages importants du texte d'Eschyle qui inaugurent de nouvelles sections de la pièce. Le f. 79 porte sur les premiers vers du second épisode (passage dialogué entre le chœur, le héraut et Clytemnestre, soit les v. 489-502 ou 467-480 dans la numérotation utilisée par Saussure). Il est à insérer entre les pages 207 et 208 de l'édition de Mejía Quijano \& Restrepo Montoya (Saussure 2008).

Le f. 80 porte sur les premiers vers du deuxième stasimon (passage chanté par le chœur, v. 681-698 ou v. 660-674 dans la numérotation utilisée par Saussure). Ce feuillet est à insérer entre les pages 224 et 225 de Saussure 2008. Un brouillon de ce feuillet est conservé dans AdS 372, f. 27 et transcrit dans Saussure $2008: 225$ et Saussure $2011: 333$.

Les deux feuillets sont d'une facture similaire à celle des manuscrits déjà connus. Ils présentent une traduction accompagnée de commentaires d'ordre linguistique ou philologique. Dans le f. 79, Saussure aborde la question débattue de l'attribution des v. 489-502 au chœur ou à Clytemnestre ${ }^{21}$. Le f. 80 quant à lui, contient la traduction de la figure étymologique sur le

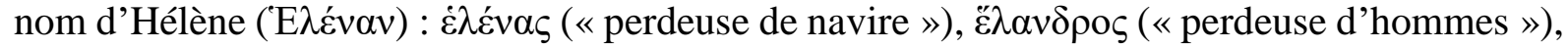
$\dot{\varepsilon} \lambda \dot{\varepsilon} \pi \tau \mathrm{\partial} \lambda \mathrm{i}$ («perdeuse de villes ») que Saussure ne commente pas ${ }^{22}$.

La transcription adopte les conventions éditoriales usuelles :

- XXX indique un texte barré

$\circ \underline{X X X}$ indique un texte souligné

- les chevrons $\langle\mathrm{XXX}\rangle$ (qu'on distinguera des parenthèses angulaires \langle\rangle utilisées par Saussure) indiquent un texte marginal, interlinéaire ou une surcharge.

- Les abréviations n'ont pas été résolues.

La transcription de chaque feuillet est précédée du texte grec commenté, dans l'édition de Mazon.

\section{Eschyle, Agamemnon, v. 489-502}

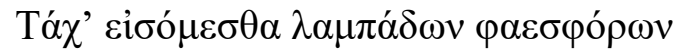

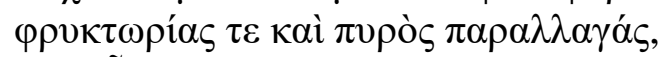

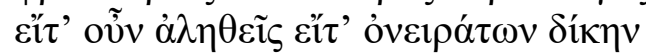

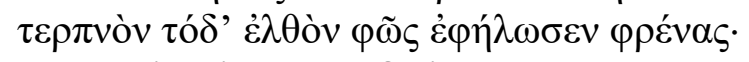

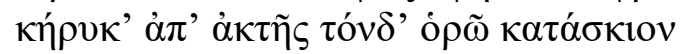

\footnotetext{
${ }^{21}$ Sur cette question, v. Dettori (1992 : 80 sq.) et Judet de la Combe (2001 : $\left.151 s q.\right)$.

${ }^{22}$ On pourra confronter les remarques de Saussure au commentaire du même passage par Judet de la Combe (1982: 10-43).
} 


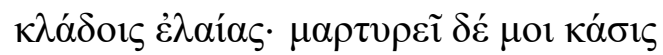

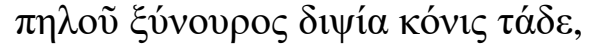

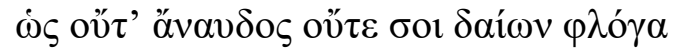

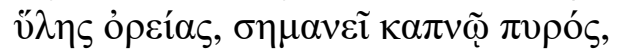

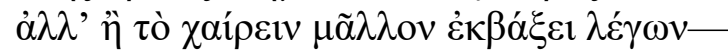

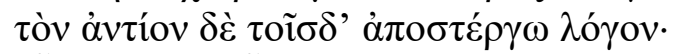

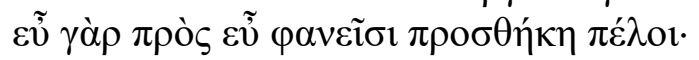

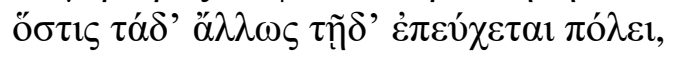

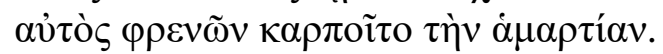

\section{[f. 79]}

466 seq.

Le chœur (ds les Mss. Clytemnestre)

Ns n'allo ne <n'allons pas> tarderens pas à savoir s'il y quelque chese <ce qu'il y a> de <véridique> dans ces transmissions de flammes, de feux, et de signaux, ou si à la manière des songes ce jeu de lumière $(\varphi \tilde{\omega} \varsigma)$, n'est venu flatter nos<tre> espérances <espoir> que pour mieux abuser nos esprits.

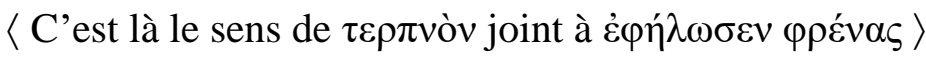

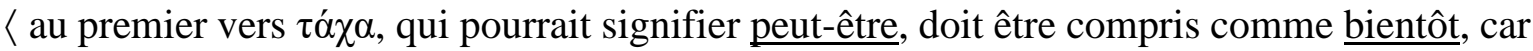

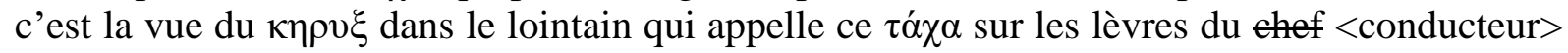
du chœur $\rangle$.

J'aperçois, venant du rivage, le messager que voilà, portant<eur> des rameaux d'olivier

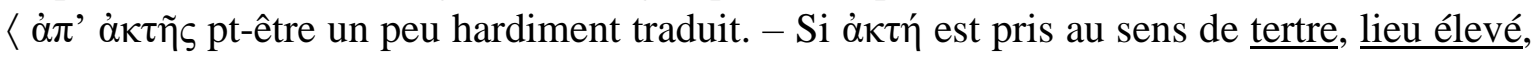
j'aperçois du haut du lieu élevé où je suis moi, chœur <mais malgré la const. cela est moins naturel>>

et la poussière brûlante qui $\langle$ sur les vêtements $\rangle$ se marie à la boue est pour moi la $\langle\mathrm{e}\rangle$ sûre preuve < garant> de ceci : que les <nouvelles> qu'il nous annoncera, il ne le $<$ s $>$ signifiera plus sans qu'on entende le son de la voix humaine, ni par la fumée de quelque feu, en allumant la flamme d'une espèce quelconque de bois des montagnes. <(Ironiquement)> Mais ou bien il nous confirmera notre sujet de joie <d'allégresse> par de d'intelligibles paroles $(\lambda \varepsilon ́ \gamma \omega v)<\mathrm{ou}$ bien $>$ (Points de suspension $)$... ou bien ... Mais assez, et je déteste toute parole contraire à un tel vœu. Notre vœu est qu'<Puisse> à de si favorables augures s'ajouter une heureuse réalité.

〈Littéralement : qu'un supplément Puisse un supplément se produire de la bonne manière en eutre <sus> de choses qui ont paru bien <qui n'ont jusqu'à présent que l'apparence de> > TS.VP

$\underline{479-480 \text { (TSVP) }}$

\section{[f. 79v]}

Ensuite paroles à mettre dans la bouche d'un des choreutes :

Quiconque forme pour cette cité un autre $<$ genre de $>$ vœu, qu'il soit seul à récolter le fruit de sen du péché de son esprit.

Schneidewin suppose que les 2 derniers vers sont prononcés par un des choreutes, et oublie le'argument principal : - que les manuscrits (mettant 467-478 ds la bouche de Clyt. mettent 479-480 dans celle du Chœur, donc connaissent un changement de persenne <d'interlocuteur> entre ces deux morceaux). En outre il méconnaît le deuxième argument et le détruit lui-même en corrigeant

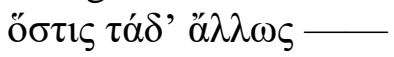

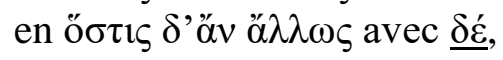

L'absence de liaison comme $\delta \varepsilon \dot{\varepsilon}$ s'explique par le fait du nouvel interloc. et si on admet ce nouvel interloc. il est difficile d'admettre $\delta \varepsilon$. 


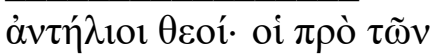

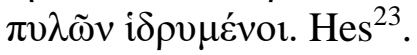

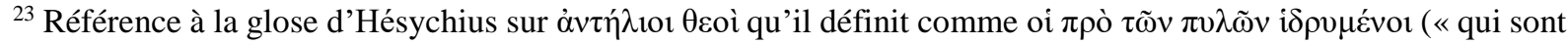
placés devant les portes »). Ce syntagme, qui figure plus loin au v. 519, Saussure le traduit ainsi : «divinités antéliennes, 〈placées devant les portes faisant face au soleil levant 〉» (Saussure 2008 : 209). 
Eschyle, Agamemnon, v. 681-698.

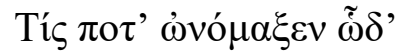

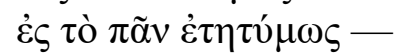

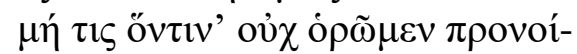

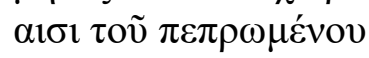

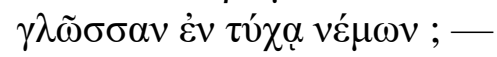

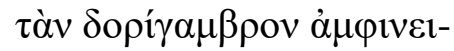

$\kappa \tilde{\eta} \theta$ ' ${ }^{\circ} \lambda \dot{\varepsilon} v \alpha \nu ; \dot{\varepsilon} \pi \varepsilon i ̀ ~ \pi \rho \varepsilon \pi o ́ v \tau \omega \varsigma$

$\dot{\varepsilon} \lambda \varepsilon \dot{\varepsilon} v \alpha \varsigma, \varepsilon \check{\varepsilon} \lambda \alpha v \delta \rho \circ \varsigma, \dot{\varepsilon} \lambda \dot{\varepsilon}-$

$\pi \tau \mathrm{\sigma} \lambda \mathrm{i \varsigma}, \dot{\varepsilon} \kappa \tau \tilde{\omega} \nu \dot{\alpha} \beta \rho о \tau i ́ \mu \omega \nu$

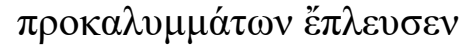

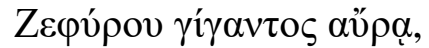

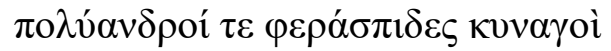

$\kappa \alpha \tau^{\prime}$ ' $\chi \chi \nu 0 \varsigma \pi \lambda \alpha \tau \tilde{\alpha} \nu$ ö $\varphi \alpha \nu \tau o v$

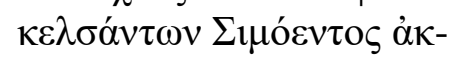

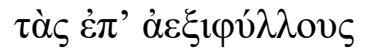

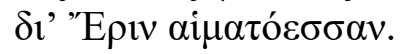

\section{[f. 80]}

Qui donc inventa le nom si inexorablement <impitoyablement> prophétique - (litt. si complètement vrai) - de cette Hélène dont la main devait être disputée au prix de la guerre entre les peuples $\langle$ résume à la fois $\delta$ opí $\gamma \alpha \mu \beta \rho o v$ et $\alpha \mu \varphi \imath v \varepsilon \imath \kappa \tilde{\eta}\rangle$. Ne serait-ce pas quelque invisible dieu ou mortel dont la langue était guidée avec justesse ( $\dot{\varepsilon} v \tau v ́ \chi \alpha$ ) par le pressentiment de la destinée de cette femme ? < litt. de ce qui était destiné > Puisqu'on pourrait dire au plus vrai sens du mot $(\pi \rho \varepsilon \pi \delta ́ v \tau \omega \varsigma)$ que c'était une Helenaus, une Helandros <ou> une Heléptolis qui de sa chambre nuptiale s'enfuyait vers l'Orient sous à la faveur du souffle gigantesque de Zephyros.

$\left\langle 1^{\circ}\right.$ Chambre nuptiale est représenté dans le texte par $\alpha \beta \rho \tau^{\prime} \mu \omega \nu \pi \rho о \kappa \alpha \lambda \nu \mu$ rideaux précieux et de fine tissu.

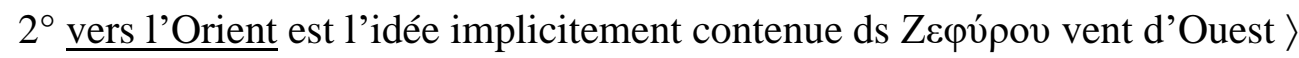

Non sans que la poursuivîssent $<t>$ à la trace $\left(\kappa \alpha \tau^{\prime}{ }^{\prime} \chi^{\prime} \chi 0 \varsigma\right)$, une armée nombreuse de chasseurs cuirassés, lorsque les fugitifs eurent mis à l'abri leur invisible nef barque sous les rives feuillees verdoyantes du Simoïs, afin qu'on en vînt à une sanglante dispute ( $\delta \imath^{\prime}$ 'épıv $\left.\alpha i \mu\right)$

$\langle 1$. "Non sans que" est dans $\tau \varepsilon$, et dans le $\varepsilon \pi \lambda \lambda \varepsilon v \sigma \alpha \nu$ qu'il faut ss-entendre après $\kappa v v \bar{\alpha} \gamma o$ í.

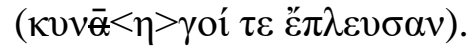

2. Le sujet de $\kappa \varepsilon \lambda \sigma \alpha ́ v \tau \omega v$ est dans tous les cas sous-entendu. C'est Paris et [f. 80 v] Hélène, les

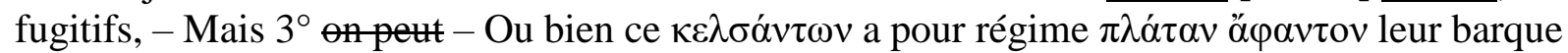
invisible (dans ce cas virgule après íl $\chi v o s$

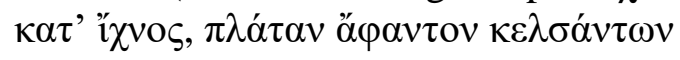

Ou bien si l'on a $\pi \lambda \alpha \tau \tilde{\alpha} v$

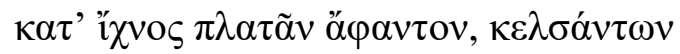

Dans ce cas a) $\kappa \varepsilon \lambda \sigma \alpha ́ v \tau \omega \nu$ est sans régime «ayant abordé aux rives du Simoïs. »b) $\pi \lambda \alpha \tau \tilde{\alpha} \nu$ $=\pi \lambda \alpha \tau \tilde{\omega} v$. c) la virgule se trouve après $\alpha$ $(\varphi \alpha v \tau o v$. d) le sens est - poursuivi à la trace invisible des vaisseaux, 


\section{BIBLIOGRAPHIE}

AMACKER, René (1994), «Correspondance Bally-Saussure», Cahiers Ferdinand de Saussure 48, p. 95-127.

AMACKER, René (2006), « Saussure et la transcription officielle du sanscrit (1894) », Cahiers Ferdinand de Saussure 59, p. 175-178.

AMACKER, René \& BOUQUET, Simon (1988), «Dix-huit notes étymologiques inédites de Ferdinand de Saussure », Cahiers Ferdinand de Saussure 42, p. 215-244.

BENVENISTE, Émile (1964), « Lettres de Ferdinand de Saussure à Antoine Meillet », Cahiers Ferdinand de Saussure 21, p. 91-130.

CHIDICHIMO, Alessandro (2009), «Les premières leçons de Saussure à Genève, 1891 : textes, témoins, manuscrits », Cahiers Ferdinand de Saussure 62, p. 257-277.

CHIDICHIMO, Alessandro (2019), «Saussure, $1896 »$, Acta Structuralica 4 : https://acta.structuralica.org/pub-228235.

DECIMO, Marc (2012), «Saussure correcteur de Louis Duvau », Genesis 35, p. 195-200.

DECIMO, Marc (2014), Sciences et pataphysique 2. Comment la linguistique vint à Paris : de Michel Bréal à Ferdinand de Saussure, Dijon, Les Presses du réel.

DETTORI, Emanuele (1992), L'Interlocuzione difficile. Corifeo dialogante nel dramma classico, Pise, Giardini.

D’OTTAVI, Giuseppe (2014), « Nine easy pieces. Les manuscrits de Ferdinand de Saussure à Harvard », in V. Chepiga \& E. Sofia (dir.), Archives et manuscrits de linguistes, Louvainla-Neuve, Academia-l'Harmattan, p. 99-132.

FLEURY, Michel (1964), « Notes et documents sur Ferdinand de Saussure (1880-1891)», Annuaires de l'École pratique des hautes études 97/1, p. 35-67.

GODEL, Robert (1960), «Inventaire des manuscrits de F. de Saussure remis à la Bibliothèque Publique et Universitaire de Genève », Cahiers Ferdinand de Saussure 17, p. 5-11.

JOSEPH, John E. (2012), Saussure, Oxford-New York, Oxford University Press.

JUDET DE LA COMBE, Pierre (1982), Agamemnon 2. Deuxième stasimon, accueil d'Agamemnon, troisième stasimon, dernier « stasimon », Lille, Presses Universitaires de Lille.

JUDET DE LA COMBE, Pierre (2001), L'Agamemnon d'Eschyle: Commentaire des dialogues, Villeneuve d'Ascq, Presses universitaires du Septentrion: http://books.openedition.org/septentrion/66964

MARCHESE, Maria Pia \& MURANO, Francesca (2015), «Ferdinand de Saussure et l'épigraphie », Cahiers Ferdinand de Saussure 68, p. 95-111.

MEJIA QUIJANO, Claudia \& RESTREPO MONTOYA, Natalia (2008), «Ferdinand de Saussure, traducteur », Cahiers Ferdinand de Saussure 61, p. 175-198.

MURANO, Francesca (2015), «Les tables d'Héraclée chez Saussure et Bally (BGE, Ms. fr. 3957 env. 7 et 5131 env. 1-2). Du maître à l'élève », Cahiers Ferdinand de Saussure 68 , p. 205-221.

MURANO, Francesca (2017), Saussure, Bally e la linguistica greca. I corsi ginevrini del 18931903, Alessandria, Edizioni dell'Orso.

PESINI, Luca (2010), «Fragments inédits de la collaboration Saussure-Bally (BGE, Ms. fr. 5134) », Cahiers Ferdinand de Saussure 63, p. 81-94.

PESINI, Luca (2012), «Un autre fragment inédit de la correspondance Saussure-Bally (BGE, Ms. fr. 5134, ff. 21-22, 29-32) », Cahiers Ferdinand de Saussure 65, p. 215-238.

PICCINI, Silvia (2020 [2021]), «La correspondance de Saussure à Niedermann (1904-1908). L'école de Saussure : admirer et pourtant ne pas comprendre », Cahiers Ferdinand de Saussure 72, p. 141-164.

PINAULT, Georges-Jean (2012), «Inédits de Ferdinand de Saussure, extraits de la 
correspondance avec Louis Havet en 1879 », Cahiers Ferdinand de Saussure 65, p. 173214.

SAUSSURE, Ferdinand de (1922), Recueil des publications scientifiques de Ferdinand de Saussure, publié par. Ch. Bally et L. Gautier, Genève, Société Anonyme des Éditions Sonor.

SAUSSURE, Ferdinand de (1990), «Le sens du mot. Ms. fr. 3970/c : un corso di morfologia indeuropea a cura di F. Angeli e C. Vallini », AIQN 12, p. 365-424.

SAUSSURE, Ferdinand de (2008), «Eschyle, Agamemnon. Traduction de Ferdinand de Saussure », édition par C. Mejía Quijano and N. Restrepo Montoya, Cahiers Ferdinand de Saussure 61, p. 199-238.

SAUSSURE, Ferdinand de (2011), Science du langage : "De la double essence du langage » et autres documents du ms. BGE Arch. de Saussure 372.Édition par R. Amacker, Genève, Droz.

SAUSSURE, Ferdinand de (2014), Une vie en lettres : 1866-1913.Édition par C. Mejía Quijano, Nantes, Cécile Defaut.

SAUSSURE, Ferdinand de (2018), La grammaire du gotique. Deux cours inédits. 1. Cours de grammaire gotique (1890-1891) 2. Cours de grammaire gothique (1881-1882) accompagnés d'autres articles de Saussure sur le gotique. Édités avec notes et commentaires par A. Rousseau, Paris, Honoré Champion.

SAVATOVSKY, Dan (1999), «Véritable grammaire comparée et grammaire véritablement comparée (Saussure à Paris) », Cahiers Ferdinand de Saussure 52, p. 169-188.

SAVATOVSKY, Dan (2003), "Comment faire école? (Saussure à Paris, II) », Cahiers Ferdinand de Saussure 56, p. 311-329.

SECHEHAYE, Albert (2009), «Phonétique du grec et du latin. Cours de Mr Ferdinand de Saussure, Université de Genève 1891-1892 », édité par A. Chidichimo, Cahiers Ferdinand de Saussure 62, p. 279-288.

TESTENOIRE, Pierre-Yves (2013), Ferdinand de Saussure à la recherche des anagrammes, Limoges, Lambert Lucas.

TESTENOIRE, Pierre-Yves (2015), «Deux nouvelles lettres de Ferdinand de Saussure à Antoine Meillet », Cahiers Ferdinand de Saussure 68, p. 189-204.

TESTENOIRE Pierre-Yves (2018), «Onze lettres (1884-1893) de Ferdinand de Saussure découvertes dans les archives de la Société de linguistique de Paris », Cahiers Ferdinand de Saussure 71, p. 161-182. 